\title{
Robust and Adaptive Excitation Signal Generation for Input and Output Constrained Systems
}

\author{
Per Hägg*, Christian A. Larsson* and Håkan Hjalmarsson*
}

\begin{abstract}
Generating signals with a prespecified autocorrelation, available from some input design, while satisfying constraints on input and output signals of the systems which the signal is to be applied on is an important problem in system identification. This paper extends a recently proposed method for such signal generation. The method is modified to be robust to uncertainties in the system model. An adaptive formulation is also given which allows for improving the input design online. The adaptive method is compared to the true optimal input design on a simulation example. The robustness properties of the method are illustrated on an experimental setup.
\end{abstract}

\section{INTRODUCTION}

$\mathbf{S}$ YSTEM identification is routinely used in industry as a tool for plant modeling. A vital part of any identification experiment is choosing the signal to be used to excite the system. A correctly chosen excitation signal can significantly improve the quality of the resulting model while, on the other hand, a badly chosen signal can result in a useless model. This has lead to the study of optimal experiment or input design for system identification.

There are many proposed methods for input design available in the literature. The common idea of all methods is based on the realization that the quality of the estimates can be influenced by the choice of the input through the covariance matrix. Early works, such as [1], [2], focused on optimizing some measure of the covariance matrix directly. Such techniques are still often employed in practice. Since the last decades, the focus has shifted to measuring quality in terms of the application of the model. This has resulted in the ideas of identification for control [3], [4], [5], least costly identification [6] and applications oriented input design [7]. In these methods, the input signal is designed in terms of its spectral properties making it difficult to find signals that satisfy time domain constrains on, for example, the input amplitude.

In practice, it is often important to limit the input and output amplitudes from the system during the experiment. The constraints on input usually come from actuator saturation while output constraints are there to keep the system in a safe operation region and to maintain product quality.

To be able to handle the time domain constraints in the input design, different approaches are possible. The first approach is to leave out the constraints during the input design and instead enforce them when the signal is realized.

\footnotetext{
The research leading to these results has received funding from the European Union's Seventh Framework Programme (FP7/2007-2013) under grant agreement $\mathrm{n}^{\mathrm{O}} 257059$, The 'Autoprofit' project (www.fp7-autoprofit.eu), the Swedish Research Council and the Linnaeus Center ACCESS at KTH.

*The authors are with the Automatic Control Lab and ACCESS, School of Electrical Engineering, KTH, SE-100 44 Stockholm, Sweden. (e-mails: \{per.hagg,christian.larsson,hakan.hjalmarsson\}@ee.kth.se).
}

One simple method to satisfy constraints on the input is to use a binary sequence [8], [9], [10]. Another possibility is to consider signals that are sums of sinusoids [11], [12]. The amplitude spectrum can easily be shaped since each sinusoid gives a peak at the corresponding frequency. The phase of the signal can then be designed such that time domain requirements, e.g., on input signal amplitude, are satisfied [13]. This often leads to a non convex optimization problem but reasonable heuristics have been proposed.

A related concept is the plant-friendly identification techniques developed in for example [14]. The focus here is to keep output and input variance low to maintain product quality and to minimize strain on actuators during the identification experiment.

Another recent approach is to do the input design in time domain. It is then straightforward to formulate the input and output constraints. However, the resulting optimization problem is non-convex but can be approximated via convex relaxations [15].

Model predictive control (MPC) is an often used control strategy for system with constraints. Recently several approaches to including excitation in the MPC formulation have been proposed. The methods in [16] and [17] focus on making the input persitently exciting. A similar technique is presented in [18], with the main difference that the final purpose of the identified model is incorporated in their formulation.

A well known problem with optimal input design is that the desired input signal depends on the unknown system, which one wants to identify. One approach to get around this is to make the design robust or insensitive to the true system [19], [20], another is to make the design adaptive, i.e., as information from the system is gathered the input signal properties are changed, see e.g., [21].

In this contribution we extend a method for designing an excitation sequence directly in time presented in [20]. The method tries to match the autocorrelation of the generated sequence to a desired autocorrelation, found for example by input design, while maintaining input and output constraints. The input signal is generated in open loop in a receding horizon fashion, calculating the input sample by sample. The method does, however, rely on perfect information of the system under consideration to be able to satisfy the output constraints.

In this paper we combine the signal generation algorithm from [20] with tools from Robust MPC [22] and adaptive input design. The main idea is to make the signal generation algorithm robust to uncertainties in the model of the system. A sample is generated and applied to the system. The resulting output sample is then used to identify a better model 
with, hopefully, less uncertainty. The new model is then used to generate a new sample and so on. As our knowledge of the system gets better we can also improve the input design similar to what is done in adaptive input design.

\section{Signal Generation}

The method we develop in this paper is based on the signal generation algorithm presented in [23], which is briefly presented in this section.

The aim is to generate $N$ samples of a signal $U_{N} \triangleq$ $\left[\begin{array}{lll}u_{1} & \ldots & u_{N}\end{array}\right]^{T}$ with prescribed autocorrelation $\mathbf{r}^{d}=$ $\left[\begin{array}{lll}r^{d}(0) & \cdots & r^{d}(n)\end{array}\right]^{T}$ while satisfying input and output constraint on the (SISO) system

$$
\begin{aligned}
x_{t+1} & =F x_{t}+G u_{t}, \\
y_{t} & =H x_{t}, \\
u_{\min } \leq u & \leq u_{\max }, \\
y_{\min } \leq y & \leq y_{\max },
\end{aligned}
$$

which the signal is to be applied to.

The (biased) sample autocorrelation of the signal $U_{t}$ is defined as

$$
r_{t}(\tau)=\frac{1}{t} \sum_{i=\tau+1}^{t} u_{i} u_{i-\tau}=\frac{t-1}{t} r_{t-1}(\tau)+\frac{1}{t} u_{t} u_{t-\tau} .
$$

The goal is to minimize the the Euclidean distance between the obtained and desired autocovariance while satisfying input and output constraints, i.e.,

$$
\begin{array}{ll}
\underset{U_{N}}{\operatorname{minimize}} & \left\|\mathbf{r}_{N}-\mathbf{r}^{d}\right\|_{2}^{2}, \\
\text { subject to } & (1),
\end{array}
$$

where $\mathbf{r}_{t}=\left[\begin{array}{lll}r_{t}(0) & \cdots & r_{t}(n)\end{array}\right]^{T}$ is the sample autocorrelation sequence of the signal $U_{N}$. The optimization problem (3) is non-convex and is in general hard to solve. Instead the following receding horizon solution to the problem was proposed in [23]. At sample $t$ solve the optimization problem

$$
\begin{array}{cl}
\underset{u_{1}}{\operatorname{minimize}} & \left\|\mathbf{r}_{t}-\mathbf{r}^{d}\right\|_{2}^{2}, \\
\text { subject to } & \hat{x}_{i+1}=F \hat{x}_{i}+G u_{i}, \\
& \hat{y}_{i}=H \hat{x}_{i}, \\
& \hat{x}_{1}=x_{t}, \\
& u_{\min } \leq \hat{u}_{1} \leq u_{\max }, \\
& y_{\min } \leq \hat{y}_{i} \leq y_{\max }, \quad i=1 \ldots N_{y} .
\end{array}
$$

where $N_{y}$ is the output horizon, i.e., how far in the future we consider that the output should satisfy the constraints. The optimal solution, $u_{1}^{\star}$, to (4) is implemented, i.e., $u_{t}=u_{1}^{\star}$, and the optimization is performed iteratively in receding horizon fashion. If $N_{y}>1$ we set $u_{2}=\cdots=u_{N_{y}}=0$, i.e., the input is considered zero over the remaining part of the output horizon. For this special case when only one input sample is considered in each iteration of (4) it was shown that the solution can be found analytically, see [23] for details. This is the case we consider in this paper.

The steps of the algorithm are:

$$
\text { 1) Set } t=1, x_{0}=x \text { and } \mathbf{r}_{0}=\mathbf{0}_{n+1} \text {. }
$$

2) Solve the optimization problem (4). If the solution is non unique, arbitrarily choose the optimum $u^{\star}$.

3) Set $u_{t}=u_{1}^{\star}$.

4) Set $x_{t+1}=F x_{t}+G u_{1}^{\star}$.

5) Update $\mathbf{r}_{t}$ according to (2).

6) If $t<N, t \leftarrow t+1$ and go to Step 2; otherwise stop.

\section{Robust Signal Generation}

The signal generation algorithm outlined in the previous section requires perfect knowledge of the system and its state. However, this contradicts the purpose of the method: to use the generated signal to identify an unknown system. This problem appears in all formulations of optimal input design. To get around this fundamental problem we will now make the algorithm robust to uncertainties in the system.

Assume that we know that the system that the signal is to be applied to lies inside some uncertainty region, i.e., the state space matrices of the system can be written as

$$
\{F(\theta), G(\theta), H(\theta)\}
$$

where $\theta=\left[\begin{array}{llll}\theta^{1} & \theta^{2} & \ldots & \theta^{n}\end{array}\right]$ is an uncertain parameter vector in the set $\Delta^{\theta}$. Furthermore, assume that the states of the system at time $t$ lie inside an uncertainty region $\Delta_{t}^{x}$. For now we consider the uncertainty regions as known. We will later discuss how these can be obtained.

Now, the robust problem formulation at time $t$ is

$$
\begin{array}{cl}
\underset{\hat{u}_{1}}{\operatorname{minimize}} & \left\|\mathbf{r}_{t}-\mathbf{r}^{d}\right\|_{2}^{2}, \\
\text { subject to } & \hat{x}_{i+1}=F(\theta) \hat{x}_{i}+G(\theta) \hat{u}_{i}, \\
& \hat{y}_{i}=H(\theta) \hat{x}_{i}, \\
& \hat{x}_{1}=x_{t}, \\
& u_{\min } \leq \hat{u}_{1} \leq u_{\max }, \\
& y_{\min } \leq \hat{y}_{i} \leq y_{\max }, i=1 \ldots N_{y}, \\
& \theta \in \Delta^{\theta}, \quad x_{t} \in \Delta_{t}^{x}
\end{array}
$$

Hence (6) requires satisfying infinitely many constraints and is therefore in general hard to solve.

\section{A. Scenario Based Optimization}

To relax the robust optimization problem (6), we use scenario based optimization [24]. The idea is to select a finite number of the original constraints to satisfy. Assume that we draw $N_{k}$ independent and identically distributed samples $\theta^{(1)}, \ldots, \theta^{\left(N_{k}\right)}$ uniformly distributed in $\Delta_{\theta}$ and similarly draw $N_{k}$ samples $x_{t}^{(1)}, \ldots, x_{t}^{\left(N_{k}\right)} \in \Delta_{t}^{x}$. The robust constrains of (6) can then be relaxed to

$$
\begin{aligned}
-y_{\max } \leq y_{t+k}^{(i)} \leq y_{\max }, \quad k & =1, \ldots, N_{y}, \\
i & =1, \ldots, N_{k},
\end{aligned}
$$

where

$$
y_{t+k}^{(i)}=H\left(\theta^{(i)}\right)\left(F^{k}\left(\theta^{(i)}\right) x_{t}^{(i)}+F^{k-1}\left(\theta^{(i)}\right) G\left(\theta^{(i)}\right) u_{t}\right) \text {. }
$$

We now have $N_{k}$ convex constraints on $u_{t}$. We note that only the most limiting upper and lower bound imposed by the constraints need to be taken into account in the optimization, hence only one upper and one lower bound on $u_{t}$ are necessary, which allows us to reduce the number of constraints. The accuracy of scenario based optimization has been studied in for example [24], [25]. 


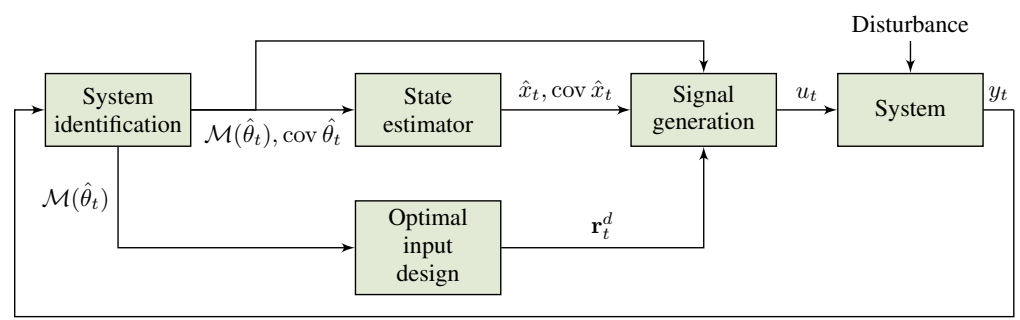

Fig. 1: Sketch of the adaptive signal generation idea

\section{Adaptive Signal Generation}

The proposed robust signal generation scheme can be quite conservative if we have large uncertainties in our prior knowledge of the system, in other words, $u_{t}$ must be small to be able to satisfy the output constraint for all possible systems in the uncertainty region. An adaptive scheme, where the model is improved as more and more data are available, can reduce this conservativeness [26], [21]. To this end, we develop an adaptive version of the proposed method in the following sections. A block diagram outlining the idea of the method is shown in Figure 1. The individual parts of steps of the method are described in further details below.

\section{A. System Identification}

Given data $\left\{y_{s}, u_{s}\right\}_{s=1}^{t}$ up to the current time $t$ the system identification block identifies a new model of the system. Given a model structure parameterized by the vector $\theta$

$$
\mathcal{M}(\theta): \quad \begin{aligned}
x_{t+1} & =F(\theta) x_{t}+G(\theta) u_{t}+v_{t} \\
y_{t} & =H(\theta) x_{t}+e_{t}
\end{aligned}
$$

where $v_{t}$ and $e_{t}$ are zero mean, Gaussian noise processes, the goal is to find an estimate, $\hat{\theta}_{t}$ of the unknown parameters $\theta$ describing the studied system. Furthermore we would like to find an estimate, $\hat{P}_{t}$ of the estimated parameter covariance matrix $\operatorname{cov}\left(\hat{\theta}_{t}\right)$.

Most standard system identification methods can be used such as for example the Prediction Error Method [27] or Subspace Identification methods [28]. Preferably, the method should be recursive to take advantage of the sample by sample real time implementation.

\section{B. State estimator}

The objective of the state estimator is to estimate the current state of the system given data up to time $t$, the current model $\mathcal{M}\left(\hat{\theta}_{t}\right)$ and the uncertainty in the parameters of the model, $\hat{P}_{t}$ from the system identification step.

For linear models with Gaussian noise, considered in this paper, the standard Kalman filter [29] can be used resulting in

$$
\begin{aligned}
\hat{x}_{s \mid s-1} & =F\left(\theta_{t}\right) \hat{x}_{s-1 \mid s-1}+B\left(\theta_{t}\right) u_{s} \\
P_{s \mid s-1} & =F\left(\theta_{t}\right) P_{s-1 \mid s-1} F^{T}\left(\theta_{t}\right)+Q \\
K_{s} & =P_{s \mid s-1} H^{T}\left(\theta_{t}\right)\left(H\left(\theta_{t}\right) P_{s \mid s-1} H^{T}\left(\theta_{t}\right)+R\right)^{-1} \\
\hat{x}_{s \mid s} & =\hat{x}_{s \mid s-1}+K_{s}\left(y_{s}-H\left(\theta_{t}\right) \hat{x}_{s \mid s-1}\right) \\
P_{s \mid s} & =P_{s \mid s-1}-K_{s} H\left(\theta_{t}\right) P_{s \mid s-1}
\end{aligned}
$$

for $s=0, \ldots, t$. Above $Q$ is the process noise covariance matrix and $R$ is the measurement noise covariance matrix.
Running the Kalman filter generates an estimate of the current state $\hat{x}_{t \mid t}$ and an estimate of the error covariance of the estimated state $P_{t \mid t}$. The matrix $Q$ can be seen as a tuning parameter of how good the used model is. A large $Q$ could be seen as if we have large process noise acting on the system and hence the model is uncertain and vice versa for a small $Q$. The question is then how do we use the knowledge of the uncertainties of the identified model in the Kalman filter? To the best of our knowledge there is no good method to transform uncertainties in the parameters to a corresponding process noise covariance $Q$.

It is well known that under mild assumptions [27] the accuracy of the identified model is improved as more data is used in the identification. To capture this in a simple way in the Kalman filter we let $Q$ decay somehow as a function of $t$. How fast $Q$ should decay depends on the application. Another way to use the uncertainty in the model could be to use a robust Kalman filter, see for example [30] and the references therein.

\section{Input Design}

The objective of the Optimal Input Design part is to find an optimal input signal that excites the system in such a way that its important properties are revealed. What these important properties are depends on the intended use of the model. Input design is often formulated as an optimization problem where one tries to minimize the experimental effort needed, such as input power or experimental length, while fulfilling some specifications on the accuracy of the identified model [6], [7]. The converse formulation is also possible: given an input power and experiment length, find the input signal that minimizes some cost function related to the identified model. The requirements on the model could for example come from the intended use of the model, where a certain accuracy is needed. The outcome of the optimization is the desired autocovariance of the input signal.

One inherent problem with input design is that the solution depends on the unknown true system. Here we solve this problem by using the identified model instead of the true system in the design. Hence, from the identified model, $\mathcal{M}\left(\hat{\theta}_{t}\right)$, of the system at time $t$, a desired autocovariance of the input signal, $r_{t}^{d}$, is calculated.

\section{Signal Generation}

The signal generation block generates an input sample to be applied to the system using the robust method derived in Section III. The desired autocorrelation of the generated signal $r_{t}^{d}$ comes either from input design, the adaptive input design or from a user choice. 
The uncertainty region of the model parameters $\theta$ is calculated from the estimated parameter vector $\hat{\theta}_{t}$ and its estimated covariance matrix $\hat{P}_{t}$ as

$$
\Delta_{\theta}=\left\{\theta \mid\left(\theta-\hat{\theta}_{t}\right)^{T} \hat{P}_{t}^{-1}\left(\theta-\hat{\theta}_{t}\right) \leq K_{\theta}\right\}
$$

where $K_{\theta}$ is some user defined constant. The motivation for this choice of uncertainty region is that under mild assumptions $\sqrt{t}\left(\hat{\theta}-\theta_{0}\right)$ is asymptotically, in the number of data, normally distributed. The vector $\theta_{0}$ is the parameters of the true underlying system. See [27] for details. By choosing $K_{\theta}=\chi_{\alpha}^{2}(n)$, where $\chi_{\alpha}^{2}(n)$ is the value of the $\chi^{2}$ distribution with probability $\alpha$ and $n=\operatorname{dim} \hat{\theta}_{t}$, (8) becomes a confident ellipsoid. Then the parameter vector, $\theta_{0}$, of the true system lies inside $\Delta_{\theta}$ with probability $\alpha$. Here we will throughout use $\alpha=0.95$, i.e., that with $95 \%$ probability the true system is within the confidence region $\Delta_{\theta}$.

Similarly the uncertainty in the estimated state is calculated from $\hat{x}_{t \mid t}$ and the state covariance matrix $P_{t \mid t}$ given by the Kalman filter as

$$
\Delta_{t}^{x}=\left\{x_{t} \mid\left(x_{t}-\hat{x}_{t \mid t}\right)^{T} P_{t \mid t}^{-1}\left(x_{t}-\hat{x}_{t \mid t}\right) \leq K_{x}\right\},
$$

with $K_{x}=\chi_{\alpha}^{2}\left(\operatorname{dim} x_{t}\right)$.

\section{E. Initialization}

Before we start the adaptive method we need to initialize all the parameters. Firstly, a model structure $\mathcal{M}(\theta)$, parameterized by the unknown parameters $\theta$ is needed. Furthermore an initial guess of $\theta$ and its uncertainty region are required. These could, for example, come from first principles modeling or from previous experience of the considered process.

Secondly, the Kalman filter needs to be initialized. This includes choosing an initial state estimate $\hat{x}_{t \mid t}$ and its uncertainty $P_{0 \mid 0}$. We also have to choose $R$, the measurement noise covariance matrix and $Q$, the process noise covariance matrix and how fast $Q$ should decay in $t$.

\section{F. Termination}

The algorithm runs until some user specified criterion is fulfilled. This could be that a certain number of samples have been generated or that the estimated parameters are accurate enough. The required accuracy of the parameters could for example come from the intended use of the model, i.e., when the model is used some performance specifications are satisfied.

\section{EXAMPLES}

In this section we evaluate the proposed algorithm on two examples. The first example studies the convergence of the algorithm when used in adaptive input design. In the second example, the method is tested experimentally on a double tank lab process with constraints on input and output signal amplitudes.

\section{A. $\mathcal{L}_{2}$-gain estimation}

This example is taken from [31]. We study the performance degradation resulting from using the proposed adaptive signal generation scheme compared to what would be obtained if the optimal autocorrelation had been know at the beginning of the experiment.
Consider the fourth order FIR-system

$y_{t}=\theta_{1} u_{t-1}+\theta_{2} u_{t-2}+\theta_{3} u_{t-3}+\theta_{4} u_{t-4}+e_{t}=G(q, \theta) u_{t}+e_{t}$.

where $e_{t}$ is zero mean white Gaussian noise with variance $\sigma_{e}^{2}$. The goal is to estimate the squared $\mathcal{L}_{2}$ gain

$$
\|G\|_{2}^{2}=\frac{1}{2 \pi} \int_{-\pi}^{\pi}\left|G\left(e^{j \omega}, \theta\right)\right|^{2} d \omega=\theta^{T} \theta .
$$

At the end of the experiment of length $N=200$ we would like to have a certain accuracy of the identified model while at the same time minimizeing the required input power.

In [31] it is shown that the autocorrelation sequence of the input signal $\mathbf{r}^{d}$ that minimizes the input power while keeping the variance of the estimated $\mathcal{L}_{2}$-gain of the system lower than $\gamma$ is given by the solution to the following optimization problem

$$
\begin{array}{cl}
\underset{\mathbf{r}^{d}}{\operatorname{minimize}} & r_{0}^{d} \\
\text { subject to } & {\left[\begin{array}{cc}
R\left(\mathbf{r}^{d}\right) & 2 \theta \\
2 \theta^{T} & \frac{\gamma N}{\sigma_{e}^{2}}
\end{array}\right] \succeq 0} \\
& \mathbf{r}^{d} \text { defines a positive spectrum, }
\end{array}
$$

where $R\left(\mathbf{r}^{d}\right)$ is the Toeplitz matrix

$$
R\left(\mathbf{r}^{d}\right)=\left[\begin{array}{ccc}
r_{0}^{d} & \cdots & r_{n}^{d} \\
\vdots & \ddots & \vdots \\
r_{n}^{d} & \cdots & r_{0}^{d}
\end{array}\right]
$$

The constraint that $\mathbf{r}^{d}$ defines a positive spectrum can be transformed into an LMI in several ways, see for instance [31], [7] for details. Hence, the optimization problem can be formulated as an SDP and can be solved efficiently.

The adaptive identification scheme is applied to this identification problem. We assume that we know the correct model structure. Since the true values of the parameter vector $\theta$ and the noise variance are unknown to us we cannot directly find the optimal input signal according to (10). Instead we use the current estimate of the parameters, $\hat{\theta}_{t}$, and an estimate of the noise variance $\hat{\sigma}_{t}^{2}$ in the optimization problem to get the desired autocorrelation at time $t, \mathbf{r}_{t}^{d}$.

To evaluate the performance of the adaptive identification scheme, 200 Monte Carlo simulations were carried out. We set $\gamma=0.001$, the number of autocovariance lag to match to $n=3$ and the parameter vector of the true system to $\theta=\left[\begin{array}{llll}0.9, & 0.6, & 0.2, & 0.3\end{array}\right]^{T}$. In this case there are no input or output constraints. This means that the state estimator is not needed in this example.

In each time step a new model is recursively identified using the Matlab command rarx [32] and the noise variance is recursively estimated as

$$
\sigma_{t}^{2}=\frac{t-1}{t} \sigma_{t-1}^{2}+\frac{1}{t}\left(y_{t}-\varphi_{t}^{T} \hat{\theta}_{t}\right)^{2}
$$

where $\varphi_{t}^{T}$ is the regressor vector used in the recursive identification. Based on the identified model, a new desired autocorrelation is found and subsequently a new input sample is generated.

Figure 2 shows the sample variance of the estimate of the squared $\mathcal{L}_{2}$-gain. The aim in the input design was to keep the 


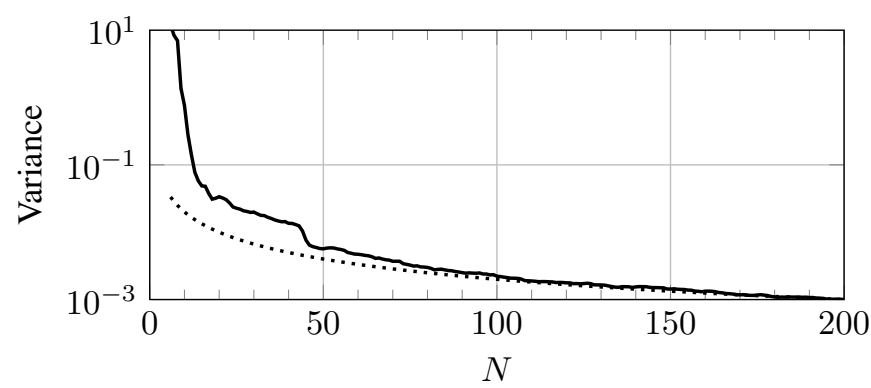

Fig. 2: Variance of the estimated $\mathcal{L}_{2}$-gain estimated from Monte-Carlo simulations ( - ) and the asymptotic variance if the optimal input was known $(\cdots \cdots)$.

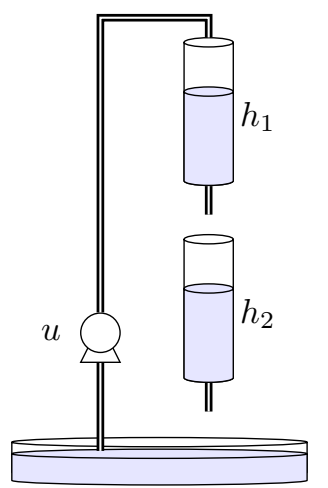

Fig. 3: The double tank process.

variance at the end below $\gamma=0.001$. The sample variance at $N=200$ is indeed very close to the desired variance. In Figure 2 we also show the theoretical asymptotic variance if we from the beginning knew the optimal input signal. After about 100 samples the variance using the adaptive signal generation is close to the variance we would get if we from the beginning knew the optimal signal.

\section{B. Identification of Water Tank System}

We apply the proposed adaptive signal generation and identification method to a double tank lab process [33]. An illustration of the process is shown in Figure 3. A pump pumps water into the upper tank. Water then flows through orifices located in the bottom of each tank, from the upper tank into the lower tank, and from the lower tank into a water basin. The input to the system is the applied voltage to the pump and the outputs are the water levels in each tank.

The objective of the experiment is to identify a linear model of the tank. The process is however nonlinear and hence we identify a linear model around a equilibrium point. To keep the state within a region where the linear approximation is good we limit the deviation from the equilibrium point to $1.5 \mathrm{~cm}$ for the upper tank and $0.5 \mathrm{~cm}$ for the lower tank. The reason for the harder constraints on the lower tank is to make the problem more interesting. If they would have been the same then the constraint of the level in lower tank would rarely be active. Furthermore we constrain the input to be within $\pm 3 \mathrm{~V}$ around the equilibrium voltage. In this case we want to excite the system with a white input signal
TABLE I: Parameters of the double tank process.

\begin{tabular}{ccc}
\hline Parameter & Nominal value \& uncertainty & Description \\
\hline$a_{1}$ & $0.17 \pm 0.035 \mathrm{~cm}^{2}$ & outlet area tank 1 \\
$a_{2}$ & $0.17 \pm 0.035 \mathrm{~cm}^{2}$ & outlet area tank 2 \\
$k$ & $3.0 \pm 0.75 \mathrm{~cm}^{3} / \mathrm{sV}$ & flow constant \\
$A$ & $15.15 \mathrm{~cm}^{2}$ & cross-section area of tanks \\
$g$ & $10^{3} \mathrm{~cm} / \mathrm{s}^{2}$ & gravitational constant \\
\hline
\end{tabular}

with variance 3 , i.e.,

$$
\begin{aligned}
r^{d}(0) & =3 \\
r^{d}(i) & =0, \quad i=1, \ldots, n,
\end{aligned}
$$

and we want to match $n=50$ lags. The sampling rate for the process is $1 \mathrm{~Hz}$ and we run the algorithm for $N=600$ samples.

1) Model: A linear model around an equilibrium point $h^{0}=\left[\begin{array}{ll}h_{1}^{0} & h_{2}^{0}\end{array}\right]^{T}$ and $u^{0}$ is given by [33]

$$
\begin{aligned}
\frac{\mathrm{d} \bar{x}_{t}}{\mathrm{~d} t} & =\left[\begin{array}{cc}
-\frac{1}{\tau_{1}} & 0 \\
\frac{1}{\tau_{1}} & -\frac{1}{\tau_{2}}
\end{array}\right] \bar{x}_{t}+\left[\begin{array}{c}
\frac{k}{A} \\
0
\end{array}\right] \bar{u}_{t} \\
y_{t} & =\bar{x}_{t}+e_{t}
\end{aligned}
$$

where $\bar{x}_{t}=h_{t}-h^{0}, \bar{u}_{t}=u_{t}-u^{0}, \tau_{i}=\frac{A}{a_{i}} \sqrt{\frac{2 h_{i}^{0}}{g}}$ and $e_{t}$ is assumed to be white zero mean Gaussian measurement noise.

2) Experiment: An initial model and the uncertainties are chosen based on previous experience of the system. The parameters and their uncertainties are shown in Table I. The equilibrium point during the experiment is $h^{0}=$ $\left[\begin{array}{ll}14.6 & 12.7\end{array}\right]^{T} \mathrm{~cm}$ and $u^{0}=4.5 \mathrm{~V}$ and are considered known exactly.

For the first 60 samples the initial model is used to generate the input signal. The Kalman filter is initialized with

$$
\begin{aligned}
& R=0.001 \cdot I, Q_{0}=\left[\begin{array}{cc}
0.05 & 0 \\
0 & 0.005
\end{array}\right] \\
& P_{0 \mid 0}=0.01 \cdot I, \quad x_{0}=\left[\begin{array}{ll}
0 & 0
\end{array}\right]^{T}
\end{aligned}
$$

After the 60 samples have been collected each new measurement from the system is used to identify a new model of the system. The model (14) is used with the unknown parameters $\theta=\left[\begin{array}{lll}a_{1} & a_{2} & k\end{array}\right]^{T}$. The parameters and their covariances are then estimated using the Matlab System Identification Toolbox command idgrey [32]. The new model is used in the Kalman filter to estimate the current state and the estimated covariance matrix is used to calculate new uncertainty regions for the parameters, see (8) and (9). To take the reduced model uncertainty into account in the Kalman filter we reduce the state covariance matrix in each time step according to

$$
Q_{t}=0.99^{t} \cdot Q_{0}
$$

A new input signal sample is calculated with the robust algorithm and applied to the process. This is repeated until 600 samples have been generated. 


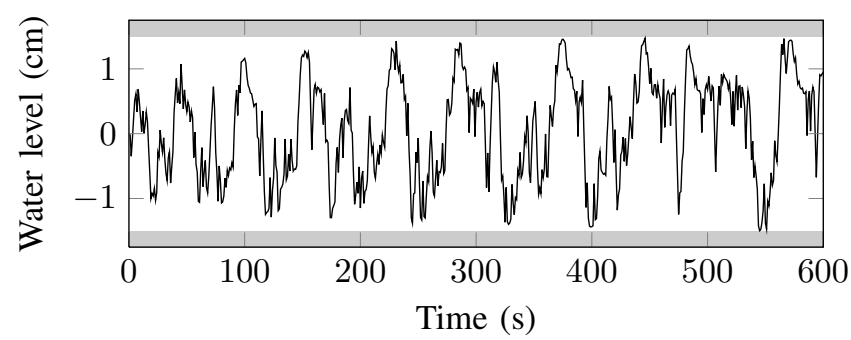

(a) Measured output $y_{1}$.

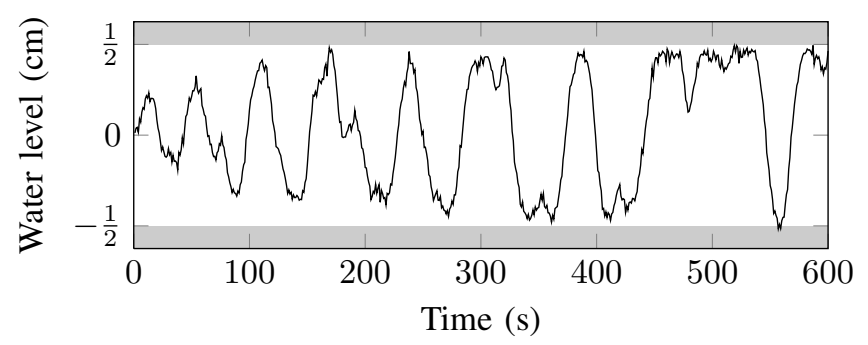

(b) Measured output $y_{2}$.

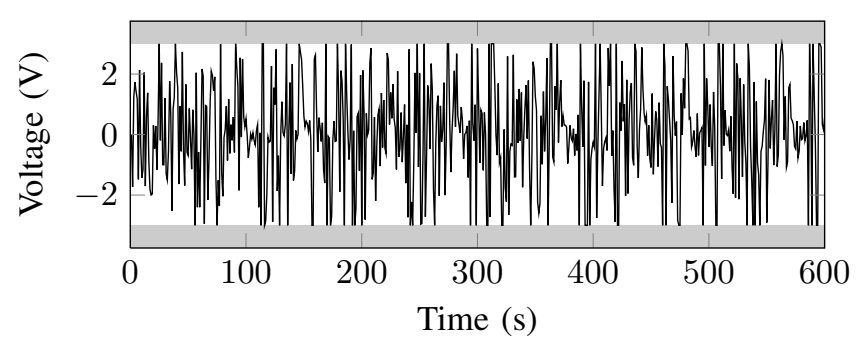

(c) Generated input signal, $u_{t}$.

Fig. 4: Measured output and generated input signal around the equilibrium point with constraints.

3) Results: The measured outputs and the generated input signal is shown in Figure 4. We see that both the input and the outputs satisfy the constraints during the experiment except for two samples around $t=560$. In the beginning of the experiment we have large uncertainties in the model and the states, and hence the algorithm is conservative. But as more data from the system is available the identified model gets better and the system can be excited more, keeping the outputs closer to their constraints. Since we do not have access to the true values of the state but only noisy measurement, it could be that the true system did not violate the constraints. However, the reason for the two samples not satisfying the constraints could stem from the fact that the identified linear model does not have exactly the same gain as the nonlinear system close to the constraints (far from the working point). To counteract this problem one could for example stop decreasing $Q_{t}$ after a certain time to take the remaining uncertainty in the model into account. Another, alternative is to add uncertainty when the state is close to the constraints.

The autocorrelation of the generated input is shown in Figure 5. The generated signal is quite close to white but the variance is 2.9 compared to the desired variance 3.0.

The estimated parameters at the end of the experiment are,

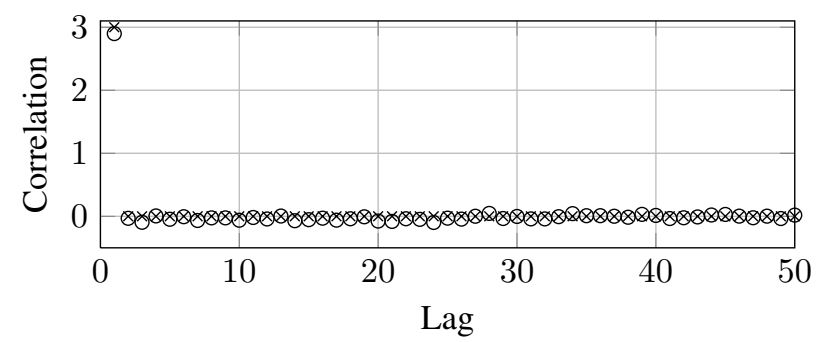

Fig. 5: Sample autocorrelation of the generated input signal (o) and desired autocorrelation ( $\times$ ).

$\hat{a}_{1}=0.142, \hat{a}_{2}=0.153$ and $\hat{k}=3.21$.

\section{CONCLUSIONS}

In this paper we presented an algorithm for recursive system identification. The goal is to excite a system with a input signal with prescribed autocovariance when the system under consideration has input and output constraints. The method is a development of the signal generation algorithm presented in [23]. The main idea was to define uncertainty regions for the system and then generate an input such that the constraints are satisfied for all possible systems within the uncertainty region. The measured output is then used to identify a better model and update the uncertainty of the model. The new model could then also be used in input design, redesigning the desired properties of the input signal in each time step. These steps are then repeated until the model is good enough.

The performance of the algorithm was verified via MonteCarlo simulations and applied to the double tank lab process with promising results. However more work should be done to study the convergence of the proposed method and analyze its performance.

The signal generation is formulated as a MPC-problem. It would be interesting to see if it is possible to improve the performance if we consider longer input horizons, i.e., not only optimizing the input one step ahead but optimize further in time.

\section{REFERENCES}

[1] V. Fedorov, Theory of optimal experiments, ser. Probability and mathematical statistics. Academic Press, 1972.

[2] G. Goodwin and R. Payne, Dynamic system identification: experiment design and data analysis, ser. Mathematics in science and engineering. Academic Press, 1977.

[3] M. Gevers and L. Ljung, "Optimal experiment design with respect to the intended model application," Automatica, vol. 22(5), pp. 543-554, 1986.

[4] M. Gevers, "Connecting identification and robust control: a new challenge," in IFAC/IFORS Symposium on identification and System Parameter Estimateion, Budapest, Hungary, 1991.

[5] H. Hjalmarsson, M. Gevers, and F. De Bruyne, "For model based control design criteria, closed loop identification gives better performance," Automatica, vol. 32, pp. 1659-1673, 1196.

[6] X. Bombois, G. Scorletti, M. Gevers, P. V. den Hof, and R. Hildebrand, "Least costly identification experiment for control," Automatica, vol. 42, no. 10 , pp. $1651-1662,2006$.

[7] H. Hjalmarsson, "System identification of complex and structured systems," European Journal of Control, vol. 15/3-4, pp. 275-310, 2009.

[8] E. Hannan, Multiple Time Series. New York: Wiley, 1970. 
[9] C. R. Rojas, J. Welsh, and G. Goodwin, "A receding horizon algorithm to generate binary signals with a prescribed autocovariance," in American Control Conference, 2007. ACC '07, july 2007, pp. 122 -127 .

[10] H. He, J. Li, and P. Stoica, Waveform Design for Active Sensing Systems: A Computational Approach. Cambridge University Press, 2012.

[11] J. Schoukens, P. Guillaume, and R. Pintelon, "Design of multisine excitations," in Control 1991. Control '91., International Conference on, mar 1991, pp. $638-643$ vol.1.

[12] R. Pintelon and J. Schoukens, System identification : a frequency domain approach. New York: IEEE Press, 2001.

[13] D. E. Rivera, H. Lee, H. D. Mittelmann, and M. W. Braun, "Constrained multisine input signals for plant-friendly identification of chemical process systems," Journal of Process Control, vol. 19, no. 4, pp. $623-635,2009$.

[14] D. E. Rivera, H. Lee, and A. Badgwell, "Plant-friendly system identification: a challenge for the process industries," in 13th IFAC Symposium on System Identification, SYSID2003, 2003.

[15] I. R. Manchester, "Input Design for System Identification via Convex Relaxation," ArXiv e-prints, Sep. 2010.

[16] G. Marafioti, "Enhanced model predictive control:dual control approach and state estimation issues," Ph.D. dissertation, Norwegian University of Science and Technology, Department of Engineering Cybernetics, 2010.

[17] J. Rathouský and V. Havlena, "Mpc-based approximation of dual control by information maximization," in Proceedings of the 18th International Conference on Process Control, M. Fikar and M. Kvasnica, Eds. Tatranská Lomnica, Slovakia: Slovak University of Technology in Bratislava, 2011, pp. 247-252.

[18] C. A. Larsson, M. Annergren, C. R. Rojas, H. Hjalmarsson, X. Bombois, A. Mesbah, and P.-E. Modén, "Model predictive control with integrated experiment design for ouput error systems," in European Control Conference, 2013, submitted. Extended version available as D3.1 Novel Algorithms for Least-costly Closed-loop Re-identification (www.fp7-autoprofit.eu)

[19] C. R. Rojas, J. S. Welsh, G. C. Goodwin, and A. Feuer, "Robust optimal experiment design for system identification," Automatica, vol. 43, no. 6, pp. 993 - 1008, 2007.

[20] C. A. Larsson, E. Geerardyn, and J. Schoukens, "Robust optimal input design," in IFAC Symposium on System Identification (SYSID 2012, Brussels), 2102, pp. 1611-1616.

[21] L. Gerencsér and H. Hjalmarsson, "Adaptive input design in system identification," in Decision and Control, 2005 and 2005 European Control Conference. CDC-ECC '05. 44th IEEE Conference on, dec. 2005, pp. $4988-4993$.

[22] J. Maciejowski, Predictive control: with constraints, ser. Pearson Education. Prentice Hall, 2002.

[23] C. A. Larsson, P. Hägg, and H. Hjalmarsson, "Generation of excitation signals with prescribed autocorrelation for input and output constrained systems," in American Control Conference, 2013. ACC 13'., 2013, submitted.

[24] G. C. Calafiore and M. C. Campi, "The Scenario Approach to Robust Control Design," IEEE Transactions on Automatic Control, vol. 51, no. 5, pp. 742-753, May 2006.

[25] M. Campi and G. Calafiore, "Notes on the Scenario Design Approach," Automatic Control, IEEE Transactions on, vol. 54, no. 2, pp. 382 385, feb. 2009.

[26] K. Lindqvist and H. Hjalmarsson, "Identification for control: Adaptive input design using convex optimization," in Conference on Decision and Control. Orlando, Florida, USA: IEEE, December 2001, pp. 4326-4331.

[27] L. Ljung, System Identification: Theory for the User, 2nd Edition. Upper Saddle River, New Jersey: Prentice Hall, 1999.

[28] P. van Overschee and B. DeMoor, Subspace Identification of Linear Systems: Theory, Implementation, Applications. Kluwer, 1996.

[29] R. E. Kalman, "A new approach to linear filtering and prediction problems," Transactions of the ASME-Journal of Basic Engineering, vol. 82, no. Series D, pp. 35-45, 1960.

[30] P. Ruckdeschel, "Optimally robust kalman filtering," Berichte des Fraunhofer ITWM, vol. 185, 2010.

[31] L. Gerencsér, H. Hjalmarsson, and J. Mårtensson, "Identification of ARX systems with non-stationary inputs asymptotic analysis with application to adaptive input design," Automatica, vol. 45, no. 3, pp. $623-633,2009$.

[32] L. Ljung, System Identification Toolbox Users Guide 2012b. The MathWorks, Inc., September 2012.
[33] K. H. Johansson, A. Horch, O. Wijk, and A. Hansson, "Teaching multivariable control using the quadruple-tank process," in Decision and Control, 1999. Proceedings of the 38th IEEE Conference on, vol. 1, 1999, pp. $807-812$ vol.1. 